\title{
Large-Eddy Simulation (LES) on the Square and Triangular Tall Buildings to Measure Drag Force
}

\author{
Abdollah B. Daemei $\left(\mathbb{D},{ }^{1}\right.$ Amiraslan Darvish $\left(\mathbb{D},{ }^{2}\right.$ Roya Aeinehvand $\left(\mathbb{D},{ }^{3}\right.$ \\ and Amirali Razzaghipour (D) $^{4}$ \\ ${ }^{1}$ Young Researchers and Elite Club, Rasht Branch, Islamic Azad University, Rasht, Iran \\ ${ }^{2}$ Faculty of Architecture and Urbanism, Imam Khomeini International University, Qazvin, Iran \\ ${ }^{3}$ School of Architecture Urban Planning Construction Engineering, Politecnico di Milano, Lecco, Italy \\ ${ }^{4}$ School of Design and the Built Environment, Curtin University, Perth, Australia \\ Correspondence should be addressed to Abdollah B. Daemei; baghaei@iaurasht.ac.ir
}

Received 1 January 2021; Accepted 3 May 2021; Published 26 May 2021

Academic Editor: Filippo Ubertini

Copyright ( $\odot 2021$ Abdollah B. Daemei et al. This is an open access article distributed under the Creative Commons Attribution License, which permits unrestricted use, distribution, and reproduction in any medium, provided the original work is properly cited.

\begin{abstract}
The wind load issues play a significant role in designing tall buildings, which has sometimes been considered an even more essential factor than earthquake loads. Also, investigating wind behavior in tall buildings is a crucial issue in architectural and structural design. A primary concern of wind engineering and aerodynamics is drag force. Drag force refers to a solid object's behavior in the relative wind flow velocity direction in terms of fluid dynamics. The investigation involved only drag forces. The Autodesk Flow Design 2014 software was utilized as a wind tunnel simulator. The Large Eddy Simulation (LES) method was used for turbulence solving. This study aims to optimize tall square and triangular-shaped buildings in order to reduce drag force under along-wind motion. For this purpose, architectural aerodynamic strategies such as chamfered, rounded, and recessed corners were applied as aerodynamic modifications. Moreover, aerodynamic forms, including tapering and setting back on shapes, were applied on 24 building models. Generally, the height $(H)$ and breadth $(b)$ ratios were set to $H: 200 \mathrm{~m}$, which is equivalent to almost 60 stories, and b: $25 \mathrm{~m}$ wide. The obtained results indicate that model S5 (with a square floor plan) achieved $0.65 C_{D}$, and the $\mathrm{t} 1$ (with a triangular floor plan) achieved $0.30 C_{D}$, which could provide the best building model to reduce drag force. In this regard, the s1 could perform over $50 \%$ better in reducing wind load. Concerning the aerodynamic modification performance, the simulation results indicate that these modifications were able to lead to over $50 \%$ better performance in reducing wind force in square samples compared to triangular samples.
\end{abstract}

\section{Introduction}

High winds are regarded to be among the most destructive natural hazards besides earthquakes. Wind loads can cause severe damage to constructions. Aerodynamic optimization is a practical approach to reduce the adverse effects of wind on tall buildings. The effect of architectural aerodynamic modification to cope with wind forces has been widely investigated, so that aerodynamic modifications (which have been known as a minor architectural treatment) are thought to be efficient, including cross-sectional shapes (horizontally) such as polygons and corners [1-6], building forms (vertically) such as tapered [7-9], setbacks [10], helical or twisted [11], and openings [12, 13].

In this context, aerodynamic forms include setback models, helical and tapered, and composite models. Besides, aerodynamic forms include recessed corners, chamfer corners, and rounded corners [14]. Wind speed is significantly notable at higher altitudes. For this reason, these types of techniques can ultimately reduce the resistance of building facades against wind effects, and finally, the adverse effect of wind on tall buildings will be lower. On the other hand, plan variation can cause the frequency of wind effect change in height. Due to a building's different geometries at 
height, vortex properties change in height, and less irritability is created by wind [15]. Furthermore, the basis of the aerodynamic building at its top method is the creation of an aerodynamic form near the top of buildings that is part of the overall aerodynamic design of the building [16].

Paying attention to the aerodynamics of the building top secures improvements regarding along-wind and across-wind building response by reducing the effect of wind-induced turbulence (vortex shedding forces) [17]. The optimum location for the along-wind openings is situated somewhere between 80 to 90 percent of the building's height [18]. The assessments in the present research found that a minimal number of studies have been conducted in Iran regarding the issue. This study proposes investigating aerodynamic forms on the models of tall buildings such as setback and tapering and compares these forms with extruded models. The main objective of this paper was to reduce drag force on building models.

In this study, a computational fluid dynamics simulation approach was conducted on 24 building models. This study proposes investigating aerodynamic forms (in terms of their architectural angles) on tall building models such as setback, tapering, and aerodynamic modifications such as chamfered, rounded, and recessed corners and compares these forms with the base model. Due to the problems mentioned above, and with the vast increase in the construction of super-tall buildings in the late $21^{\text {st }}$ century, designers and scientist have been increasingly considering a large number of approaches to mitigate wind load, owing to the fact that it is the most prominent factor to consider on the first stage of design. The significance of effective facilitation of the decreased wind impact on tall buildings became immediately apparent, especially due to the fact that architectural design has become an interdisciplinary design knowledge regarding this field.

Determining the acceptable sway limits of tall buildings is an important topic that has undergone extensive research. Controlling the lateral loads of tall and flexible buildings may raise construction cost, derived from reinforcing or strengthening the structure. Despite the cost of construction, bearing in mind the impact of wind on tall buildings, this method can lead to the reduction of structural requirements, and it can be an adequate response in reaction to various frequencies of wind motions. On the other hand, in many cases, the approach must be uniquely and specifically adapted in order to meet the features and requirements of the construction site, which ultimately causes an increase in the construction budget. This primary objective of this paper is to reduce drag force on the building models in order to address the problems mentioned earlier and the crucial issues regarding the architecture of buildings. In this study, a computational fluid dynamics simulation approach has been conducted on 24 building models.

\section{A Brief Summary of Previous Studies}

Several studies have been conducted in this research area. For instance, Lin et al. [19] studied wind flow characteristics of aerodynamic forces acting on high-rise buildings. Nine samples of building with various sections were scrutinized in a wind tunnel. Huang et al. [20] conducted a study evaluating wind forces on tall steel buildings using computational fluid dynamic techniques. Besides, concerning tall buildings' aerodynamic design carried out from different points of view [21-25], including wind pressure coefficient and wind effect at the pedestrian level [26-28], a large number of studies have been conducted regarding tall buildings, aerodynamic effects, building structure, and environmental design, from which the most relevant have been addressed in the present study. In their paper, Luo et al. [29] investigated three actuating methods that were developed to study wind turbine wakes. The numerical method generating turbulence was proposed. The results show a decent agreement with those in experiments, based on which the flow fields in the wake of a wind turbine at two tip speed ratios are examined in detail through the three actuator methods of ADM, ADM-R, and ALM.

Moreover, Rastegarian and Sharifi [30] conducted a study in terms of structural system design. In this regard, the primary objective is to interpret the probable dependency of structural performance level, and its corresponding interstory drift in conventional RC moment frames. For this purpose, interstory drift (as a dependent variable) and other structural characteristics such as the strength of materials, structural dimensions, and internal loads (as independent variables) have been taken into account. In the following, Razavi Alavi et al. [31] scrutinized an industrial doublecyclone in the HDPE drying process via a mathematical method using the ANSYS Fluent software. Zhang et al. [32] conducted a full investigation on the use of all the existing reliability methods in order to analyze high-rise buildings considering wind load.

According to this issue, porches and balconies provide the best solutions for maintaining weather balance conditions in summer and winter, and providing climate comfort. Furthermore, $\mathrm{Xu}$ and $\mathrm{Xie}$ [33] conducted a study to assess the across-wind effects on a tall building with corner modifications, including chamfered and recessed corners, and the aerodynamic forms of tapering, stepping, and twisting. Giappino et al. [34] studied a building corner aerodynamic optimization procedure aimed at decreasing the wind effect based on the surrogate model. Accordingly, wind forces and pressure distributions were measured. Kumar et al. [35] studied an experimental approach to evaluate wind pressure imposed on all four rectangular faces of a tall building. Furthermore, wind pressure, drag coefficient, lift, and torsional effects were considered along with the models' wind direction measurement. Besides, in the same way, in their study, Mou et al. [36] investigated wind pressure distributions around tall squaredshaped buildings by various ratios (HW and HT) based on computational fluid dynamics techniques. In line with that, Zhao and $\mathrm{He}$ [37] carried out a research on the characteristics of surface pressure coefficients over tall oval-shaped buildings that analyzed the effects of height-width ratio and heightthickness ratio on mean wind pressure coefficients of building surfaces. They concluded that reducing the height-thickness ratio increases the absolute values of wind pressure coefficients on building surfaces. 
The study carried out by Zhang and $\mathrm{Li}$ [38] aimed to explore wind loads on supertall buildings through wind tunnel testing and field measurement, including the investigation of natural frequencies, damping ratios, and wind-induced structural responses. Wahrhaftig and Silva [39] conducted a study to compute the drag coefficient using computational fluid dynamics on a typical tall residential building. Bairagi and Dalui [40] carried out a study to analyze and compare two setback buildings, and pressure, force, and the torsional effect were also evaluated. Besides, Quan et al. [41] studied the wind pressure and high-frequency force balance on rigid models of an actual supertall building with vertical ribs protruding from its facades. The findings indicate that vertical ribs notably reduce the most negative suction coefficients in the corners of facades and increase the overall mean and fluctuating along-wind aerodynamic forces. Kumar and Kumar [42] have conducted a study on wind pressure distribution utilizing computational fluid dynamics. Fifteen various wind incidence angles were simulated, which range from 0 to 180 degrees at an interval of 30 degrees. Zhi et al. [43] proposed a new inverse method for evaluating wind effects on tall buildings. This study investigated the effects of crucial factors such as crosssection shapes of a building, the wind-induced response type, errors of structural modal parameters, and covariance matrix of noise, using a detailed parametric study.

According to the research literature and studies that have been carried out, most research works in the field of wind aerodynamic have only focused on the effects of turbulences, wind pressure, and structural design, and only a few papers take into account the geometric and shapes design in terms of architectural science, which means that this aspect of the scientific work requires more in-depth investigation. The current paper attempts to provide an all-around form that considers both the aerodynamic form and modifications on two different types of buildings including square and triangular models.

\section{Reducing Wind Force on Tall Buildings}

In terms of aerospace or fluid mechanics science, there are several forces that wind can produce, and they all have various impacts on objects (buildings in this case), the most apparent of which are motion and wind aerodynamics that can be considered in construction in terms of drag force. Drag force is the name given to the physics and fluid dynamics of forces used to prevent objects from moving within a fluid [41]. According to the definition of Hall [42], drag is a mechanical force produced by the contact between a solid object and a fluid (which can be either liquid or gas). To generate drag force, the solid body should be in contact and touch with the fluid. If there is no fluid, there will also be no force behind it. Besides, the drag depends on the fluid properties, and also on the object's size, shape, and velocity. Equation (1) can be expressed as follows:

$$
F_{D}=\frac{1}{2} \rho v^{2} C_{D}
$$

At sea level and at $15^{\circ} \mathrm{C}$, air has a density of approximately $1.225 \mathrm{~kg} / \mathrm{m}^{3}$ (International Standard Atmosphere).

In fluid dynamics, the drag coefficient, usually defined as $\left(C_{D}, C x\right.$, and $\left.C w\right)$, is a dimensionless quantity used to determine the drag or resistance of an object to fluid spaces such as air or water. In Equation (1), the lower drag coefficient indicates that the body has less aerodynamic elongation. Therefore, it can be stated that the drag coefficient is always associated with a certain level [44]. Equation (2) mathematically defines this quantity of the drag coefficient:

$$
C_{D}=\frac{2 F_{D}}{\rho v^{2} A} .
$$

Consequently, the drag force is a vector number that includes both size and direction. It can be assumed that drag is aerodynamic friction, and one of the factors that cause the formation of a drag is the surface friction between the air and the surface of a solid object. Since friction is the interaction between solids and fluid, the magnitude of friction depends on the properties of both states of matter, solid and fluid [45]. In simple terms, this force is referred to as viscosity in fluid mechanics.

Aerodynamic optimization can be referred to as "aerodynamic modifications" and "aerodynamic forms." Architectural modifications include corner modifications that do not significantly alter the existing architectural design. Modifications to the corner, such as recessing, chamfering, and rounding, reduce the building's across wind response compared with an original building shape with sharp corners. In a prismatic building, recessed (notched), chamfered (cut), and rounded corners (see Figure 1) can reduce the building's along-wind and across-wind response by a significant amount $[46,47]$. An altered/modified corner, which reduces the building's width by 10 percent compared to a sharp corner one, the building's along-wind response by 40 percent, and the building's across-wind response by 30 percent [48].

Additionally, taking benefit of aerodynamic forms is an effective architectural strategy to reduce wind force in tall buildings, and they are divided into subgroups such as tapered, setback, and helical/twisted forms. These forms are more efficient than other shapes. For tall buildings with a circular plan (footprint), the wind force is approximately $20 \%$ less than that of buildings with a rectangular floor plan [49]. An elliptical building also behaves similarly to a circular building. According to a study carried out by Schueller [50], wind force can be reduced by up to $27 \%$ in tall buildings with elliptical designs (Figure 2).

\section{Simulation Details and Analysis Methods}

The Autodesk Flow Design 2014 software was utilized for the numerical analysis and wind tunnel simulation. Flow Design runs a transient, incompressible flow solver that uses a finite volume method. Similar to other computational fluid dynamics models, turbulence was solved using the standard Smagorinsky Large Eddy Simulation (LES) model with $\mathrm{Cs}=0.12$ [11]. Accordingly, in wind engineering, LES is 


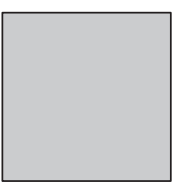

Sharp

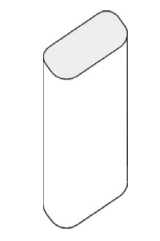

Soften corners
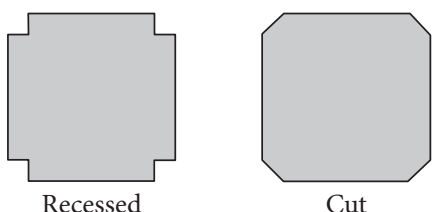

Cut

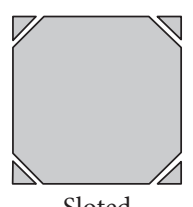

Sloted

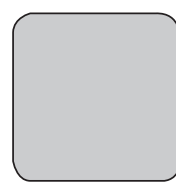

Rounded

FIgURE 1: Aerodynamic modifications.
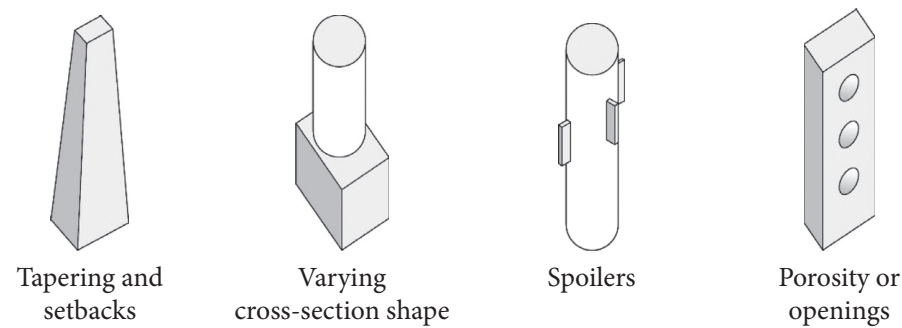

Figure 2: Aerodynamic forms.

more applicable and preferred to Reynolds-averaged Navier-Stokes (RANS) [51]. However, Flow Design has been developed to be extremely geometry tolerant, and unlike other software such as ANSYS Fluent, it does not require geometry "clean-up" in terms of meshing [52]. Its meshing technology has been developed to accept model geometry from the most widely used design packages. It accommodates solid and surface three-dimensional (3D) models and is not sensitive to small geometry imperfections [53]. For validation purposes, the results were compared with similar research papers with the same objectives and methodology after the completion of the simulation outputs.

Moreover, in order to evaluate Flow Design, the Autodesk software corporation has made various simulations and compared them with the actual empirical tests. According to their final report entitled "Flow Design Preliminary Validation Brief," the focal aspect of this software is based on machines and architectural studies. The research revealed an offset error of $6 \%$ from this software compared to experimental tests and computational simulations done by Fluent, making the software results acceptable. The models used in this study were all drawn in AutoCAD 3D volumetric definition, which is the standard and efficient approach to create 3D models and enables users to have the option to import a wide range of file formats such as STL and $3 \mathrm{DM}$. The authors of the present research used the STL file format [54].

These simulated wind loads were utilized to estimate the responses of a tall building to along-wind, which are less narrow-banded processes, based on the state-space variable approach. To establish appropriate numerical models and obtain accurate computed results, many parameters such as the calculation domain, the gird type, the algorithm, the solver type, the spatial scheme, and the time scheme are to be considered. In Autodesk Flow Design, the model wind tunnel is transparently divided into a grid of small pieces named "voxels." For the calculations, the software transparently performs this operation by automatically determining the voxel sizes based on the model under investigation and the wind tunnel. In this software, users can control the domain size, wind direction, wind velocity, and resolution level. The resolution of the analysis grid, which controls the voxels' size, was set to $200 \%$ from a possible range of $50 \%$ to $400 \%$, where higher values indicate a more detailed, but time-consuming simulation. In this study, the time scale is set to be 30 seconds (in Autodesk Flow Design, the time scale is obtained automatically when the model is imported, and the wind tunnel is configured), and the analysis type is $2 \mathrm{D}$. Based on the previous study [55], the wind tunnel domain specifications were set at X: $49 \mathrm{~B}, \mathrm{Y}$ : $25 \mathrm{~B}$, and Z: $24 \mathrm{~B}$ [53] using the approaching flow as the inflow boundary condition.

4.1. Outlining the Simulation Models. Two building models (square and triangular models) were created after careful configuration in order to investigate the influence of building dimensions on their wind characteristics. Two architectural scenarios are considered for reducing drag coefficient including aerodynamic forms (major modification) and are divided into three groups, including extruded (sharp corners), setback, and tapered. Also, aerodynamic modifications (minor modification) include rounded, chamfered, and recessed corners compared with sharp corners. Square and triangular shapes are evaluated as base forms. In general, the height and breadth ratios were set to $H \sim 200$ (about 60 stories) and $B=25 \mathrm{~m}$ wide, respectively. The proportion of major and minor aerodynamic modification models in the form is $\mathrm{b} / \mathrm{B} 1 / 20$ and $\mathrm{b} / \mathrm{B} 1 / 10$, respectively. The specifications for these models are shown in Tables 1 and 2 .

The full-scale height, total mass, and the volume of each building model are commonly about $140000.0000 \mathrm{~m} 3$. This study seeks to provide the optimal shape within the real design stage. On this basis, the volume of the building is kept the same. The geometric scale of the wind tunnel models is set at $1 / 1000$. The Reynolds number Re is based on the mean wind velocity at roof height UH, and the Square Model B's width is $\operatorname{Re}=1.9 \times 104$. Re is defined as 
TABLE 1: Schematic of building models and model IDs.

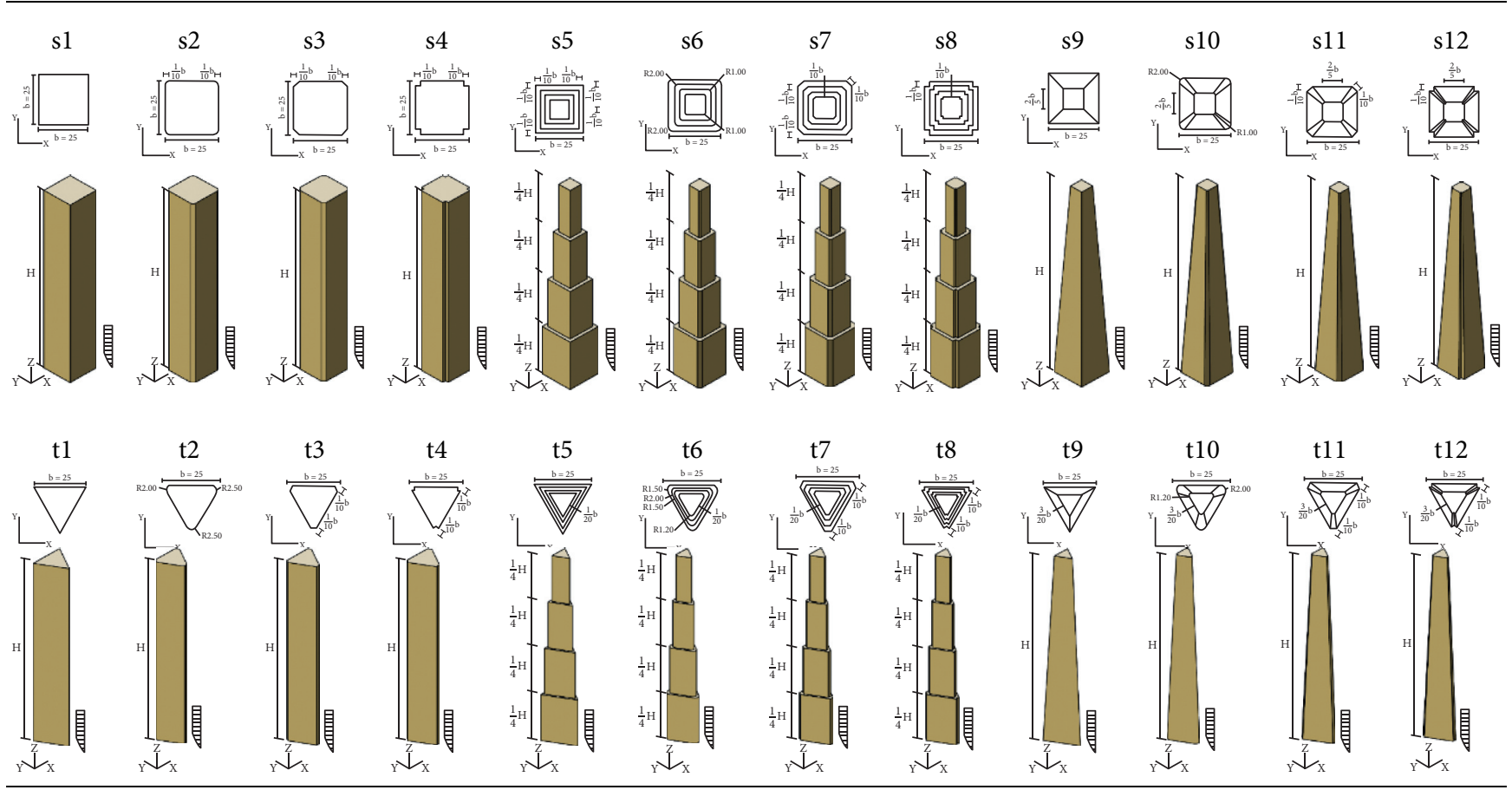

TABLE 2: Models geometry specifications.

\begin{tabular}{|c|c|c|c|c|c|c|}
\hline & Models IDs & Height $(m)$ & Cross-sectional area $\left(\mathrm{m}^{2}\right)$ & Number of sides & Length of one side ${ }^{d}$ & Internal angle $\left(^{\circ}\right)$ \\
\hline \multirow{12}{*}{ Square } & s1 & \multirow{12}{*}{200} & 675.3322 & 4 & $26(1)$ & \multirow{12}{*}{90} \\
\hline & s2 & & 674.6621 & 8 & $20.9(0.8)$ & \\
\hline & s3 & & 672.0592 & 8 & $20.9(0.8)$ & \\
\hline & s4 & & 673.5197 & 16 & $20.9(0.8)$ & \\
\hline & s5 & & & 20 & & \\
\hline & s6 & & $\mathrm{H1} 1-10229885$ & 32 & & \\
\hline & s7 & & $\mathrm{HI}=1022.9885$ & 32 & $\mathrm{H} 1=32(1.23)$ & \\
\hline & s8 & & $\mathrm{H} 2=828.6207$ & 48 & $\mathrm{H} 2=29(1.11)$ & \\
\hline & s9 & & $\mathrm{H} 3=654.7126$ & 4 & $\mathrm{H} 3=25.5(0.98)$ & \\
\hline & s10 & & $\mathrm{H} 4=501.2644$ & 8 & $\mathrm{H} 4=22.3(0.84)$ & \\
\hline & s11 & & $\mathrm{H} 5=368.2759$ & 8 & $\mathrm{H} 5=19.18(0.7)$ & \\
\hline & s12 & & & 12 & & \\
\hline \multirow{12}{*}{ Triangular } & $\mathrm{t} 1$ & \multirow{12}{*}{200} & 674.9440 & 3 & $39.50(1.51)$ & \multirow{12}{*}{60} \\
\hline & $\mathrm{t} 2$ & & 672.2385 & 6 & $39.80(1.53)$ & \\
\hline & $\mathrm{t} 3$ & & 671.4115 & 6 & $26.20(\sim 1)$ & \\
\hline & $\mathrm{t} 4$ & & 667.8165 & 9 & $26.50(\sim 1)$ & \\
\hline & $\mathrm{t} 5$ & & & 12 & & \\
\hline & t6 & & & 24 & & \\
\hline & $\mathrm{t} 7$ & & $\mathrm{H} 1=955.5849$ & 24 & $\mathrm{H} 1=47(1.8)$ & \\
\hline & t8 & & $\mathrm{H} 2=797.2596$ & 36 & $\mathrm{H} 2=42.9(1.65)$ & \\
\hline & $\begin{array}{l}10 \\
\text { t9 }\end{array}$ & & $\mathrm{H} 3=653.2644$ & $\begin{array}{c}50 \\
3\end{array}$ & $\mathrm{H} 3=38.8(1.5)$ & \\
\hline & $\begin{array}{c}\text { t9 } \\
\text { t10 }\end{array}$ & & $\mathrm{H} 4=523.4593$ & 3 & $\mathrm{H} 4=34.7(1.3)$ & \\
\hline & $\begin{array}{l}\mathrm{t} 10 \\
\mathrm{t} 11\end{array}$ & & $\mathrm{H} 5=408.2115$ & $\begin{array}{l}6 \\
6\end{array}$ & $\mathrm{H} 5=30.7(1.18)$ & \\
\hline & $\mathrm{t} 12$ & & & $\begin{array}{c}0 \\
12\end{array}$ & & \\
\hline
\end{tabular}

$$
\operatorname{Re}=\frac{\rho u L}{\mu}=\frac{u L}{v}
$$

where $\rho$ is the density of the fluid (SI units: $\mathrm{kg} / \mathrm{m}^{3}$ ), $u$ is the flow speed $(\mathrm{m} / \mathrm{s}), L$ is a characteristic linear dimension $(\mathrm{m}), \mu$ is the dynamic viscosity of the fluid (Pa.s or $\mathrm{N} \cdot \mathrm{s} / \mathrm{m}^{2}$ or $\mathrm{kg} /(\mathrm{m} \cdot \mathrm{s})$ ), and $v$ is the kinematic viscosity of the fluid $\left(\mathrm{m}^{2} / \mathrm{s}\right)$. The profiles of wind speed and turbulence intensity are presented in Figure 3.
The wind velocity and turbulence intensity at the top of the model are approximately $\mathrm{Uh}=19.7 \mathrm{~m} / \mathrm{s}$ and $\mathrm{IUH}=1.3 \mathrm{~m} / \mathrm{s}$, respectively. The profile used throughout most tests has the characteristics of a small town, forests, and features an extremely rough and uneven terrain. On this basis, the roughness class and length are 3 and 0.4 , respectively. Figure 3 presents the definitions of wind forces, moments, and the coordinate system employed 


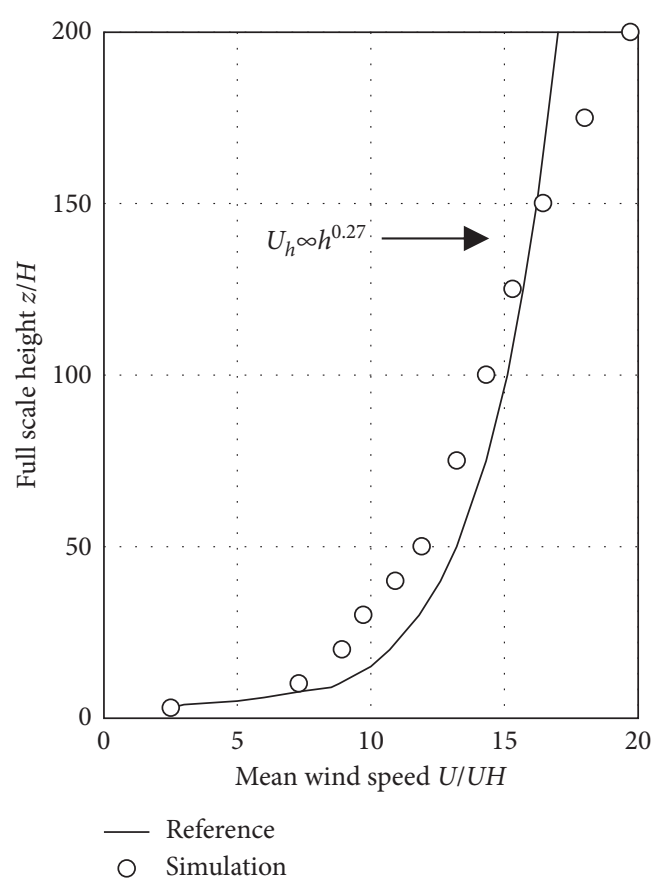

(a)

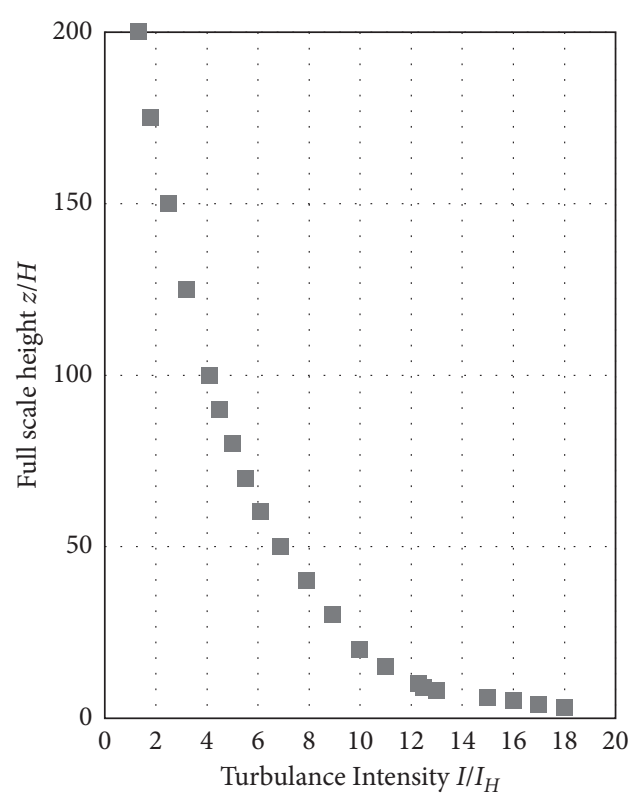

(b)

FIgURE 3: (a) Wind velocity profiles. (b) Turbulent intensity.

throughout this study. The following Research Framework Flowchart has been provided in order to help better understand the process carried out throughout the present study (Figure 4).

\section{Large-Eddy Simulation}

5.1. Aerodynamic Force Measurements. The conditions of the numerical simulation are as follows:

The downstream boundary is a convective boundary condition

The lateral and upper surfaces of the computational domain are zero gradient conditions

The spatial difference scheme is the combined use of the $2^{\text {nd }}$ and $4^{\text {th }}$ order centered difference scheme [56]

The applied algorithm is the SMAC method [11]

The entire computational domain's size in the vertical, lateral, and flow directions depends on the area that is to be represented, and the boundary conditions used. For LES, one additional requirement regarding the computational domain's overall size is that it is large enough to contain the largest, and most energetically relevant flow structures. Vertical extension of the domain for single buildings at the top of the computational domain is considered to be $3 \mathrm{H}$ above the roof of the building, where $\mathrm{H}$ is the building height. After choosing the computational domain height, the lateral extension of the domain was determined by the required blockage. Based on wind tunnel modelling guidance, the maximum blockage is suggested to be below 10\% [57].

It should be noted that the use of the LES turbulence model is highly influenced by the numerical mesh number, spatial resolution, and time scale. The Flow Design solver utilizes a Large Eddy Simulation (LES) turbulence model in order to account for the turbulence within the wind tunnel [58]. This simulation evaluated the drag coefficient $\left(C_{D}\right)$ on the 24 building models and compared them with the plot of the drag coefficient variation. Wind direction $\alpha$ is $45^{\circ}$ and $60^{\circ}$ in square and triangular models with the base being situated at the center of the models, which is normal to the building corners, depending on the building configuration. It can be stated that the effect of wind on models with square and triangular forms displays a significant difference.

Figure 5 shows the critical three points and behavior of wind effects on tall triangular and square-shaped buildings such as the wetted area, wind shear layer, and wake region. In this regard, each one of these points and responses has its specific investigation as well. The wetted area examines the building's specific surface, which is directly opposite to the wind flow and has the maximum wind pressure on it. The wake is the region of disturbed flow (often turbulent) downstream of a solid body moving through a fluid, and it is caused by the flow of the fluid around the body. In sharp corners, the body (bluff body) typically produces a separate flow, but they are not the only cause of separation, of which this separation is generally produced at the sides of buildings, called the shear layer, and generally has the maximum wind velocity. Figure 6 compares these models.

Figure 6 displays model s4 with an extruded form and sharp corners that has the highest $C_{D}$ (1.03) compared to other square models. Besides, model s3 features an extruded form and chamfered corners (0.89), s7 with a setback aerodynamic form and chamfered corners (0.87), s2 with an extruded form and chamfered corners (0.85), s8 with a setback aerodynamic form and recessed corners 


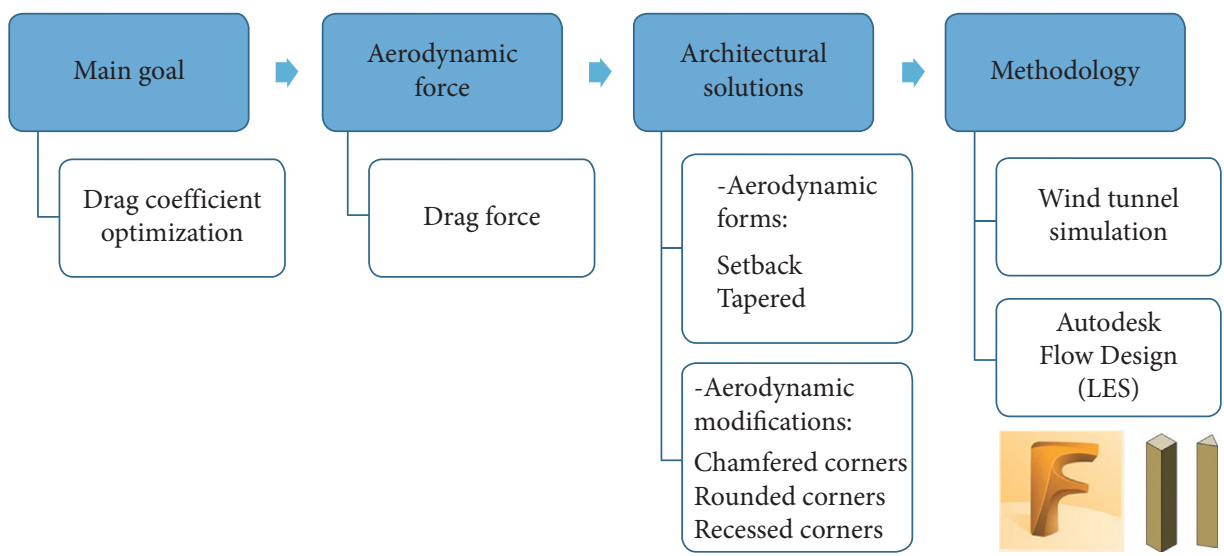

FIGURE 4: Research framework flowchart.
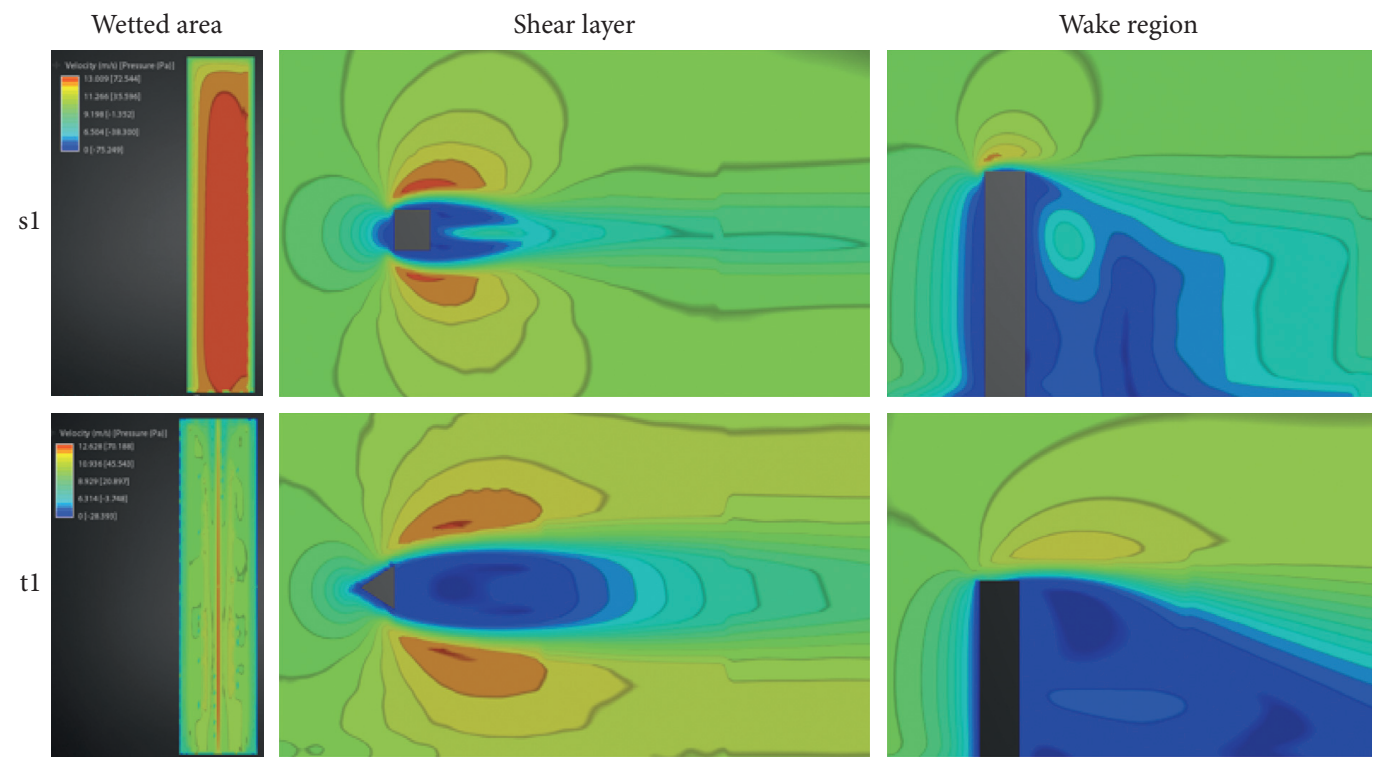

FIGURE 5: Examining the three behaviors of wind against tall buildings on the based model.

(0.84), s12 with a tapered form and recessed corners, and s10 with a tapered form and rounded corners (0.83). In the following, model s6 with a setback form and rounded corners (0.77), s9 with a tapered form and sharp corners, s11 with a tapered form and chamfered corners $(0.70), \mathrm{s} 1$ with an extruded form and sharp corners (0.66), and, finally, s5 with a setback form and sharp corners $(0.65)$ have the lowest $C_{D}$ in the models with square forms.

The $\mathrm{t} 10$ model with its tapered form and rounded corners has the highest $C_{D}(0.58)$ compared to the other triangular models. Additionally, we have 111 and t12, respectively, with a tapered form and chamfered corners, and a tapered form and recessed corners (0.57), t6 with a setback form and rounded corners (0.53), $\mathrm{t} 5$ with a setback form and sharp corners (0.50), t3 with an extruded form and chamfered corners (0.48), $t 4$ and $t 8$, respectively, with an extruded form and recessed corners, and a setback form and recessed corners (0.47). Furthermore, $\mathrm{t} 7$ with a setback form and chamfered corners (0.45), t2 with an extruded form and rounded corners $(0.41)$, t 9 with a tapered form and sharp corners (0.38), and finally t1 with its extruded form and sharp corners $(0.30)$ have the lowest $C_{D}$ among the triangular forms in this study. In Figure 7, the effect of aerodynamic techniques was evaluated concerning the reduction of $C_{D}$ for models with square and triangular forms.

Figure 7 indicates that, for square-shaped models, the tapered aerodynamic technique, and for triangular-shaped models, the extruded aerodynamic technique have been more optimal than other techniques aimed at reducing the $C_{D}$ under wind forces. Besides, Table 3 and Figure 8 indicate the impact of aerodynamic modifications, which have been evaluated regarding reducing the $C_{D}$ for models with square and triangular forms.

Table 3 and Figure 8 show that square models with sharp corners (s1, s5, and s9) have a lower drag coefficient in comparison to triangular models with sharp corners $(\mathrm{t} 1, \mathrm{t} 5$, and $t 9$ ), as well as the square models with aerodynamic modifications and rounded corners (s2, s6, and s10) compared with the triangular models with sharp corners $(t 2, t 6$, and t10), square models with aerodynamic modifications 


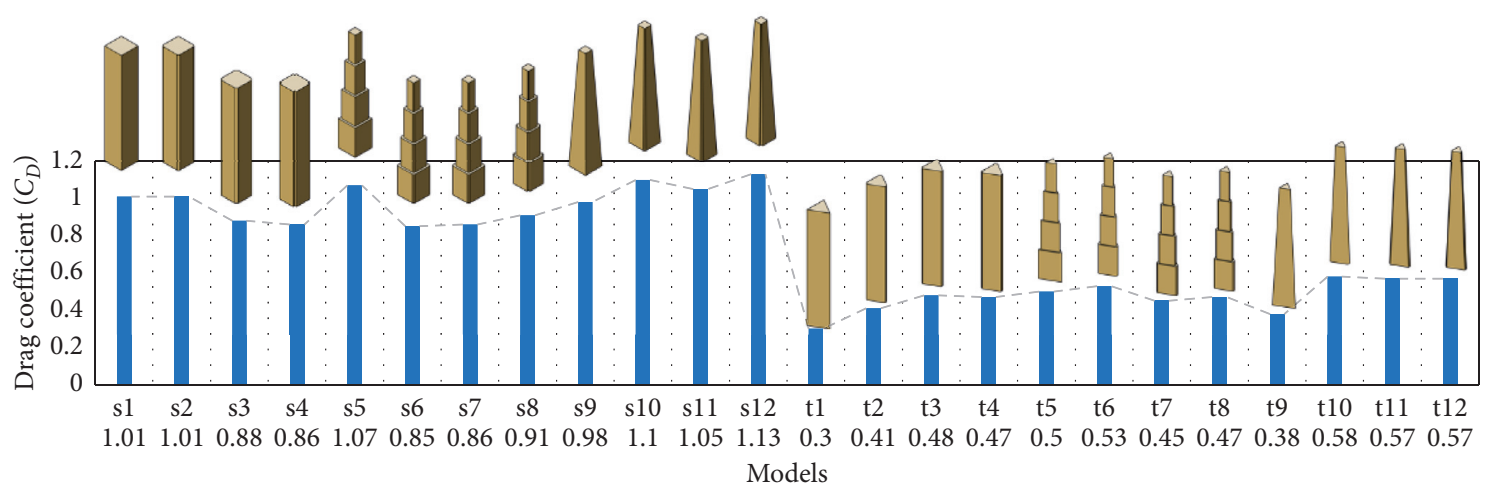

Figure 6: Comparison of models in relation to $\mathrm{C}_{D}$.

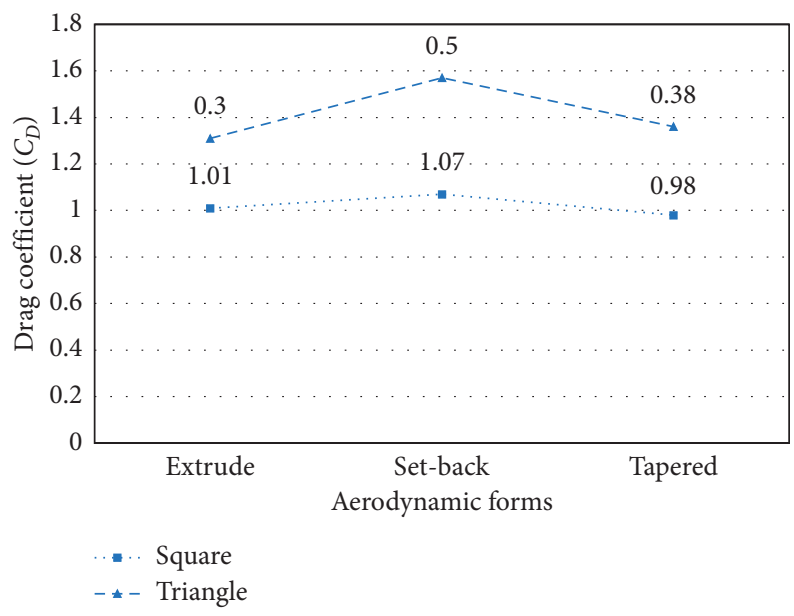

Figure 7: Comparison of the aerodynamic forms in relation to $C_{D}$.

TABLE 3: Comparison of the aerodynamic modifications in relation to $C_{D}$.

\begin{tabular}{|c|c|c|c|c|c|c|c|c|c|c|c|c|c|c|c|}
\hline \multicolumn{4}{|c|}{ Sharp } & \multicolumn{4}{|c|}{ Rounded corner } & \multicolumn{4}{|c|}{ Chamfered corner } & \multicolumn{4}{|c|}{ Recessed } \\
\hline \multicolumn{2}{|c|}{ Square } & \multicolumn{2}{|c|}{ Triangle } & \multicolumn{2}{|c|}{ Square } & \multicolumn{2}{|c|}{ Triangle } & \multicolumn{2}{|c|}{ Square } & \multicolumn{2}{|c|}{ Triangle } & \multicolumn{2}{|c|}{ Square } & \multicolumn{2}{|c|}{ Triangle } \\
\hline S1 & 0.21 & $\mathrm{~T} 1$ & 0.3 & S2 & 0.25 & $\mathrm{~T} 2$ & 0.41 & S3 & 0.25 & T3 & 0.48 & S4 & 0.29 & $\mathrm{~T} 4$ & 0.47 \\
\hline S5 & 0.14 & T5 & 0.5 & S6 & 0.16 & T6 & 0.53 & S7 & 0.17 & T7 & 0.45 & S8 & 0.17 & T8 & 0.4 \\
\hline S9 & 0.15 & T9 & 0.38 & S10 & 0.17 & $\mathrm{~T} 10$ & 0.58 & S1 & 0.14 & $\mathrm{~T} 11$ & 0.57 & S1 & 0.168 & $\mathrm{~T} 12$ & 0.5 \\
\hline \multicolumn{16}{|c|}{ Average $C_{D}$} \\
\hline \multicolumn{2}{|c|}{0.17} & \multicolumn{2}{|c|}{0.39} & \multicolumn{2}{|c|}{0.19} & \multicolumn{2}{|c|}{0.50} & & & \multicolumn{2}{|c|}{0.50} & \multicolumn{2}{|c|}{0.21} & \multicolumn{2}{|c|}{0.50} \\
\hline
\end{tabular}

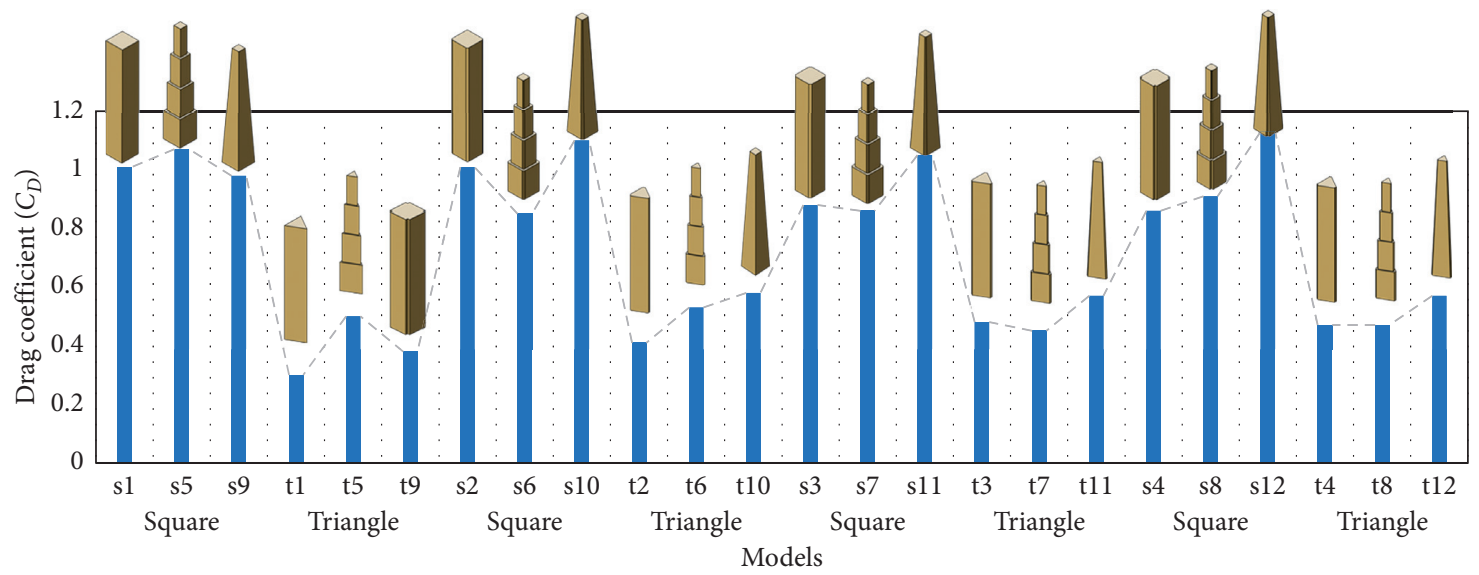

FIgURE 8: Effects of aerodynamic modifications on reducing $C_{D}$. 
and chamfered corners (s3, s7, and s11) compared with the triangular models with chamfered corners ( $\mathrm{t} 3, \mathrm{t} 7$, and $\mathrm{t} 11)$, and the square models with an extruded form. Furthermore, setback and tapered forms with recessed corners (s4, s8, and s12) were compared with the triangular models with extruded, setback, and tapered forms that have recessed corners. Models $\mathrm{t} 4, \mathrm{t} 8$, and $\mathrm{t} 12$, respectively, have a better optimal performance regarding the reduction of $C_{D}$.

In line with the results of this paper, the findings of another study that was conducted by Kawai [6] showed that, among the three modifications for corners, which include being chamfered, rounded, and recessed, a rounded corner form has a significant effect on reducing the overall wind effect. As a result, if the $\mathrm{B} / \mathrm{b}$ rate of rounding percentage is approximately $0.05 \%$, it can be assumed to be ideal for square-shaped buildings. In the following year, Tamura and Miyagi [22] carried out a study regarding aerodynamic forces on square-shaped buildings that had aerodynamic modifications. The results indicated that rounded corners could decrease the drag force and wake region. A research finding conducted by Elshaer et al. [58] showed that if the chamfered corner accounts for $20 \%$ of the building width, it could reduce wind impact on tall buildings by approximately $30 \%$.

\section{Conclusion}

The present study has examined 24 tall square and triangular-shaped buildings in order to ameliorate drag force and coefficient under along-wind motion. The research findings led to the extruded form with sharp corners, the setback form that had sharp corners, and the tapered form with sharp corners, and likewise for the triangular-shaped models. In the following, the extruded form that had sharp corners, the setback form modified using an aerodynamic technique that had chamfered corners, and the form that had been altered utilizing a tapered aerodynamic technique and had sharp corners were capable of providing a more effective optimal performance for the square-shaped models and triangular-shaped models in terms of reducing the drag coefficient. The wind tunnel simulation results ultimately indicated that the tapered aerodynamic form is more suitable for square-shaped buildings, and the extruded aerodynamic form is more appropriate for triangular-shaped buildings.

On the other hand, aerodynamic modifications such as implementing a sharp corner, a chamfered corner, a rounded corner, and a recessed corner are more appropriate for square-shaped buildings, as well as aerodynamic modifications such as implementing modifications such as a sharp, chamfered, recessed, and rounded corner for triangular-shaped buildings, respectively, with regard to modelling and reducing the drag coefficient. For this reason, it is suggested that, in order to reduce the effect of wind on 60story tall buildings with a height of approximately $200 \mathrm{~m}$, the tapered aerodynamic form can be suitable for tall square buildings, and the extruded form featuring sharp corners is ideal for tall triangular buildings. Therefore, the s5 in the square sample $\left(0.65 C_{D}\right)$ and the $t 1$ in the triangle sample $\left(0.30 C_{D}\right)$ could be considered as the best building models in terms of reducing the drag force. In this regard, the 1 model achieved over $50 \%$ better performance in reducing overall wind load. Concerning aerodynamic modification performance, the simulation results indicated that these modifications were able to bring about over 50\% better performance in reducing wind force in square samples in comparison with triangular samples.

\section{Data Availability}

The simulated data used to support the findings of this study are included within the article.

\section{Conflicts of Interest}

The authors declare that they have no conflicts of interest.

\section{References}

[1] N. Shiraishi, M. Matsumoto, H. Shirato, H. Ishizaki, M. Osada, and T. Matsui, "On aerodynamic stability effects for bluff rectangular cylinders by their corners cut," in Proceedings of the 19th National Symposium on Wind Engineering, pp. 193-198, Dayton, Ohio, May 1986.

[2] K. C. S. Kwok, P. A. Wilhelm, and B. G. Wilkie, "Effect of edge configuration on wind-induced response of tall buildings," Engineering Structures, vol. 10, no. 2, pp. 135-140, 1988.

[3] H. Hayashida and Y. Iwasa, "Aerodynamic shape effects on tall building for vortex induced vibration," Journal of Wind Engineering \& Industrial Aerodynamics, vol. 33, no. 2, pp. 237-242, 1990.

[4] H. Hayashida, Y. Mataki, and Y. Iwasa, "Aerodynamic damping effects of tall building for a vortex induced vibration," Journal of Wind Engineering \& Industrial Aerodynamics, vol. 43, no. 3, pp. 1973-1983, 1992.

[5] T. Amano, "The effect of corner-cutting of three-dimensional square cylinders on vortex-induced oscillation and galloping in uniform flow," Journal of Structural and Construction Engineering (Transactions of AIJ), vol. 60, no. 478, pp. 63-69, 1995.

[6] H. Kawai, "Effect of corner modifications on aeroelastic instabilities of tall buildings," Journal of Wind Engineering and Industrial Aerodynamics, vol. 74, no. 76, pp. 719-729, 1998.

[7] K. R. Cooper, M. Nakayama, Y. Sasaki, A. A. Fediw, S. Resende-Ide, and S. J. Zan, "Unsteady aerodynamic force measurements on a super-tall building with a tapered cross section," Journal of Wind Engineering and Industrial Aerodynamics, vol. 72, pp. 199-212, 1997.

[8] Y. C. Kim, J. Kanda, and Y. Tamura, "Wind-induced coupled motion of tall buildings with varying square plan with height," Journal of Wind Engineering and Industrial Aerodynamics, vol. 99, no. 5, pp. 638-650, 2011.

[9] Y.-M. Kim and K.-P. You, "Dynamic responses of a tapered tall building to wind loads," Journal of Wind Engineering and Industrial Aerodynamics, vol. 90, no. 15, pp. 1771-1782, 2002.

[10] Y.-M. Kim, K.-P. You, and N.-H. Ko, "Across-wind responses of an aeroelastic tapered tall building," Journal of Wind Engineering and Industrial Aerodynamics, vol. 96, no. 9, pp. 1307-1319, 2008.

[11] H. Tanaka, Y. Tamura, K. Ohtake, M. Nakai, Y. C. Kim, and E. Kumar Bandi, "Aerodynamic and flow characteristics of tall buildings with various unconventional configurations," 
International Journal of High-Rise Buildings, vol. 2, no. 3, pp. 213-228, 2013.

[12] R. Dutton and N. Isyumov, "Reduction of tall building motion by aerodynamic treatments," Journal of Wind Engineering and Industrial Aerodynamics, vol. 36, pp. 739-747, 1990.

[13] K. Miyashita, J. Katagiri, O. Nakamura et al., "Wind-induced response of high-rise buildings Effects of corner cuts or openings in square buildings," Journal of Wind Engineering and Industrial Aerodynamics, vol. 50, pp. 319-328, 1993.

[14] H. Tanaka, Y. Tamura, K. Ohtake, M. Nakai, and Y. Chul Kim, "Experimental investigation of aerodynamic forces and wind pressures acting on tall buildings with various unconventional configurations," Journal of Wind Engineering and Industrial Aerodynamics, vol. 108, pp. 179-191, 2012.

[15] J. Xie, "Aerodynamic optimization in super-tall building designs," in Proceedings of the Seventh International Colloquium on Bluff Body Aerodynamics and its Applications Shanghai, Shanghai, China, September 2012.

[16] A. Baskaran and A. Kashef, "Investigation of air flow around buildings using computational fluid dynamics techniques," Engineering Structures, vol. 18, no. 11, pp. 861-875, 1996.

[17] P. H. K. Ho, Economics Planning of Super Tall Buildings in Asia Pacific Cities Strategic Integration of Surveying Services FIG Working Week, 2007.

[18] H. Kikitsu, Y. Okuda, M. Ohashi, and J. Kanda, "POD analysis of wind velocity field in the wake region behind vibrating three-dimensional square prism," Journal of Wind Engineering and Industrial Aerodynamics, vol. 96, no. 11, pp. 2093-2103, 2008.

[19] N. Lin, C. Letchford, Y. Tamura, B. Liang, and O. Nakamura, "Characteristics of wind forces acting on tall buildings," Journal of Wind Engineering and Industrial Aerodynamics, vol. 93, no. 3, pp. 217-242, 2005.

[20] S. Huang, Q. S. Li, and S. Xu, "Numerical evaluation of wind effects on a tall steel building by CFD," Journal of Constructional Steel Research, vol. 63, no. 5, pp. 612-627, 2007.

[21] T. Tamura, T. Miyagi, and T. Kitagishi, "Numerical prediction of unsteady pressures on a square cylinder with various corner shapes," Journal of Wind Engineering and Industrial Aerodynamics, vol. 76, pp. 531-542, 1998.

[22] T. Tamura and T. Miyagi, "The effect of turbulence on aerodynamic forces on a square cylinder with various corner shapes," Journal of Wind Engineering and Industrial Aerodynamics, vol. 83, no. 3, pp. 135-145, 1999.

[23] J. C. Hu, Y. Zhou, and C. Dalton, "Effects of the corner radius on the near wake of a square prism," Experiments in Fluids, vol. 40, no. 1, pp. 106-118, 2006.

[24] P. Mendis, T. Ngo, N. Haritos, and A. Hira, "Wind loadings on tall buildings," Electronic Journal of Structural Engineering, Special Issue: Loading on Structures, vol. 41, pp. 1-14, 2007.

[25] M. Gu, P. Huang, L. Tao, X. Zhou, and Z. Fan, "Experimental study on wind loading on a complicated group-tower," Journal of Fluids and Structures, vol. 26, pp. 1142-1154, 2010.

[26] Y. Tominaga, A. Mochida, R. Yoshie et al., "AIJ guidelines for practical applications of CFD to pedestrian wind environment around buildings," Journal of Wind Engineering and Industrial Aerodynamics, vol. 96, no. 11, pp. 1749-1761, 2008.

[27] T. Stathopoulos, Wind and comfort. EACWE 5 Florence, 2009.

[28] H. Mittal, A. Sharma, and A. Gairola, "A review on the study of urban wind at the pedestrian level around buildings," Journal of Building Engineering, vol. 18, pp. 154-163, 2018.

[29] X. Luo, Q. Li, S. Xiong, and Z. Liu, "Numerical study of the wake flow of a wind turbine with consideration of the inflow turbulence," Civil Engineering Journal, vol. 4, no. 8, pp. 1907-1916, 2018.

[30] S. Rastegarian and A. Sharifi, "An investigation on the correlation of inter-story drift and performance objectives in conventional RC frames," Emerging Science Journal, vol. 2, no. 3, pp. 140-147, 2018.

[31] S. A. Razavi Alavi, L. E. Nemati, and Z. S. Alizadeh Makhmali, "A CFD study of industrial double-cyclone in HDPE drying process," Emerging Science Journal, vol. 2, no. 1, pp. 31-38, 2018.

[32] Y. Zhang, K. Yan, and T. Cheng, "Reliability analysis of high rise building considering wind load uncertainty," Civil Engineering Journal, vol. 4, no. 3, pp. 469-477, 2018.

[33] Z. Xu and J. Xie, "Assessment of across-wind responses for aerodynamic optimization of tall buildings," Wind and Structures, vol. 21, no. 5, pp. 505-521, 2015.

[34] S. Giappino, L. Rosa, G. Tomasini, and A. Zasso, "An aerodynamic and aeroelastic experimental study on a sectional and three-dimensional rectangular tall building," The Structural Design of Tall and Special Buildings, vol. 25, no. 3, pp. 139-157, 2016.

[35] S. H. Kumar, S. S. Rajan, J. A. Andrew, R. G. Babu, S. N. K. Rao, and G. J. Jawahar, "Aerodynamic coefficients for a rectangular tall building under sub-urban terrain using wind tunnel," Asian Journal of Civil Engineering, vol. 17, no. 3, pp. 325-333, 2016.

[36] B. Mou, B.-J. He, D.-X. Zhao, and K.-w. Chau, "Numerical simulation of the effects of building dimensional variation on wind pressure distribution," Engineering Applications of Computational Fluid Mechanics, vol. 11, no. 1, pp. 293-309, 2017.

[37] D.-X. Zhao and B.-J. He, "Effects of architectural shapes on surface wind pressure distribution: case studies of oval-shaped tall buildings," Journal of Building Engineering, vol. 12, pp. 219-228, 2017.

[38] J. W. Zhang and Q. S. Li, "Wind tunnel test and field measurement study of wind effects on a 600-m-high super-tall building," The Structural Design of Tall and Special Buildings, vol. 26, no. 17, Article ID e1385, 2017.

[39] A. M. Wahrhaftig and M. A. Silva, "Using computational fluid dynamics to improve the drag coefficient estimates for tall buildings under wind loading," The Structural Design of Tall and Special Buildings, vol. 27, no. 3, Article ID e1442, 2017.

[40] A. K. Bairagi and S. K. Dalui, "Comparison of aerodynamic coefficients of setback tall buildings due to wind load," Asian Journal of Civil Engineering, vol. 19, no. 2, pp. 205-221, 2018.

[41] Y. Quan, F. Hou, and M. Gu, "Effects of vertical ribs protruding from facades on the wind loads of super high-rise buildings," Wind and Structures, vol. 24, no. 2, pp. 145-169, 2017.

[42] I. G. Currie, Fundamental Mechanics of Fluids, 1974.

[43] N. Hall, http://www.grc.nasa.gov/www/k-12/airplane/drag1. html, 2015.

[44] B. W. McCormick, Aerodynamics, Aeronautics, and Flight Mechanics, John Wiley \& Sons, New York, NY, USA, 1979.

[45] S. Kumar, R. V. YNVM, and P. R. Kumar, "Aerodynamic studies of non - circular high - rise buildings," International Journal of Engineering Research in Mechanical and Civil Engineering, vol. 12, no. 5, pp. 14-21, 2015.

[46] A. Mochida and I. Y. F. Lun, "Prediction of wind environment and thermal comfort at pedestrian level in urban area," Journal of Wind Engineering and Industrial Aerodynamics, vol. 96, no. 10-11, pp. 1498-1527, 2008. 
[47] B. Blocken, J. Carmeliet, and T. Stathopoulos, "CFD evaluation of wind speed conditions in passages between parallel buildings-effect of wall-function roughness modifications for the atmospheric boundary layer flow," Journal of Wind Engineering and Industrial Aerodynamics, vol. 95, no. 9-11, pp. 941-962, 2007.

[48] Y. Kim and J. Kanda, "Characteristics of aerodynamic forces and pressures on square plan buildings with height variations," Journal of Wind Engineering and Industrial Aerodynamics, vol. 98, no. 9, pp. 449-465, 2010.

[49] B. S. Taranath, Wind and Earthquake Resistant Buildings: Structural Analysis and Design, CRC Press, Boca Raton, Florida, USA, 2004.

[50] W. Schueller, High-Rise Building Structures, John Wiley \& Sons, New York, NY, USA, 1977.

[51] D. Kumar and S. K. Dalui, "Effect of internal angles between limbs of cross plan shaped tall building under wind load," Wind and Structures, vol. 24, no. 2, pp. 95-118, 2017.

[52] L. Zhi, P. Yu, J. Tu, B. Chen, and Y. Li, "A Kalman filter based algorithm for wind load estimation on high-rise buildings," Structural Engineering \& Mechanics, vol. 64, no. 4, pp. 449459, 2017.

[53] Y. Tamura and A. Kareem, Advanced Structural Wind Engineering, Springer, Heidelberg, Germany, 2013.

[54] R. Avini, P. Kumar, and S. J. Hughes, "Wind loading on highrise buildings and the comfort effects on the occupants," Sustainable Cities and Society, vol. 45, pp. 378-394, 2019.

[55] Autodesk, Flow Design Preliminary Validation Brief. http:// www.simhub.autodesk.com/resources/flow-design-windtunnel-validation-brief, 2014.

[56] Y. Morinishi, T. S. Lund, O. V. Vasilyev, and P. Moin, "Fully conservative higher order finite difference schemes for incompressible flow," Journal of Computational Physics, vol. 143, no. 1, pp. 90-124, 1998.

[57] P. Westbury, L. Roche, B. Pierce, and J. Smith, "The effect of adjacent buildings and topographical features on the wind pressure field around buildings, BRE for the Health and Safety Executive," Research Report, vol. 157, p. 17, 2003.

[58] A. Elshaer, G. Bitsuamlak, and A. El Damatty, "Enhancing wind performance of tall buildings using corner aerodynamic optimization," Engineering Structures, vol. 136, pp. 133-148, 2017. 